\title{
Vimentin Is Involved in Peptidylarginine Deiminase 2-Induced Apoptosis of Activated Jurkat Cells
}

\author{
Pei-Chen Hsu ${ }^{1,2}$, Ya-Fan Liao ${ }^{3}$, Chin-Li Lin ${ }^{4}$, Wen-Hao Lin ${ }^{1}$, Guang-Yaw Liu ${ }^{5, *}$, and Hui-Chih Hung ${ }^{1, *}$
}

Peptidylarginine deiminase type 2 (PADI2) deiminates (or citrullinates) arginine residues in protein to citrulline residues in a $\mathrm{Ca}^{2+}$-dependent manner, and is found in lymphocytes and macrophages. Vimentin is an intermediate filament protein and a well-known substrate of PADI2. Citrullinated vimentin is found in ionomycin-induced macrophage apoptosis. Citrullinated vimentin is the target of anti-Sa antibodies, which are specific to rheumatoid arthritis, and play a critical role in the pathogenesis of the disease. To investigate the role of PADI2 in apoptosis, we generated a Jurkat cell line that overexpressed the PADI2 transgene from a tetracycline-inducible promoter, and used a combination of 12-0-tetradecanoylphorbol-13-acetate and ionomycin to activate Jurkat cells. We found that PADI2 overexpression reduced the cell viability of activated Jurkat cells in a dose- and time-dependent manner. The PADI2-overexpressed and -activated Jurkat cells presented typical manifestations of apoptosis, and exhibited greater levels of citrullinated proteins, including citrullinated vimentin. Vimentin overexpression rescued a portion of the cells from apoptosis. In conclusion, PADI2 overexpression induces apoptosis in activated Jurkat cells. Vimentin is involved in PADI2-induced apoptosis. Moreover, PADI2-overexpressed Jurkat cells secreted greater levels of vimentin after activation, and expressed more vimentin on their cell surfaces when undergoing apoptosis. Through artificially highlighting PADI2 and vimentin, we demonstrated that PADI2 and vimentin participate in the apoptotic mechanisms of activated $\mathrm{T}$ lymphocytes. The secretion and

${ }^{1}$ Department of Life Sciences and Institute of Genomics and Bioinformatics, National Chung-Hsing University, and Agricultural Biotechnology Center (ABC), National Chung Hsing University, Taiwan, Republic of China, ${ }^{2}$ Department of Medicine, Da-Chien General Hospital, Taiwan, Republic of China, ${ }^{3}$ Department of Applied Chemistry, Chaoyang University of Technology, Taiwan, Republic of China, ${ }^{4}$ Institute of Medicine, Chung Shan Medical University, ${ }^{5}$ Institute of Microbiology and Immunology, Chung Shan Medical University, and Division of Allergy, Immunology, and Rheumatology, Chung Shan Medical University Hospital, Taiwan, Republic of China

${ }^{*}$ Correspondence: liugy@csmu.edu.tw (GYL); hchung@dragon.nchu. edu.tw $(\mathrm{HCH})$

Received 30 November, 2013; revised 20 April, 2014; accepted 21 April, 2014; published online 22 May, 2014

Keywords: apoptosis, peptidylarginine deiminase type 2, vimentin surface expression of vimentin are possible ways of autoantigen presentation to the immune system.

\section{INTRODUCTION}

Peptidylarginine deiminases (PADIs; EC 3.5.3.15) deiminate positively charged arginine residues in protein to neutral citrulline residues in a $\mathrm{Ca}^{2+}$-dependent manner. To date, 5 isotypes of PADIs (PADI types 1-4 and type 6) have been found, all of which display extensive mutual sequence homologies. The greatest difference between them is their tissue-specific expression (reviewed by Vossenaar et al., 2003). PADI type 2 (PADI2) is the most widely expressed type, and is observed in skeletal muscle, the brain, the spleen, macrophages, and lymphocytes (Asaga et al., 2001; Foulquier et al., 2007; Vossenaar et al., 2004). PADI4 is expressed mainly in granulocytes (Asaga et al., 2001; Hagiwara et al., 2002; Nakashima et al., 2002) and monocytes (Nakashima et al., 1999; Vossenaar et al., 2004b). PADI4 has a nuclear localization sequence, and has been found to be present in the cell nucleus (Nakashima et al., 2002). It targets nuclear proteins, including histones and nucleophosmin/B23 (Hagiwara et al., 2002; Nakashima et al., 2002).

This posttranslational modification (deimination or citrullination) triggers a reduction in the net charge of the substrates, a loss of potential ionic bonds, interference with $\mathrm{H}$ bonds, and unfolding (Tarcsa et al., 1996). It may exert a substantial impact on the structure and function of proteins. Citrullination can also lead to increased proteolytic susceptibility. For example, citrullinated myelin basic protein is prone to be digested by aspartyl protease cathepsin D (Nicholas, 2011; Pritzker et al., 2000), citrullinated filaggrin and alpha enolase are prone to be digested by calpain-1 (Jang et al., 2012; Kamata et al., 2009), and citrullinated vimentin is prone to be digested by calpains (reviewed by Zhou and Ménard, 2002). Vimentin, a well-known substrate of PADI2 (Hojo-Nakashima et al., 2009), is an intermediate filament protein that is widely expressed in all mesenchymal cells and tissues (Hay, 1989). As in other intermediate filaments, the vimentin network spreads from the nucleus to the plasma membrane. It plays a critical role in maintaining cell and tissue integrity (Lundkvist et al., 2004).

Numerous autoimmune and neurodegenerative diseases are associated with citrullination, such as rheumatoid arthritis (RA) (De Rycke et al., 2005; Foulquier et al., 2007), multiple sclerosis (Moscarello et al., 2007; Nicholas et al., 2004), Alzheimer 
disease (Ishigami et al., 2005), and Parkinson disease (Nicholas, 2011). RA is an autoimmune disease characterized by chronic symmetric destructive polyarthritis of the peripheral joints. Among the autoantibodies found in patients with RA, autoantibodies against citrullinated proteins such as anticitrullinated protein antibodies (ACPAs) are most specific for RA Citrullinated vimentin is the target of anti-Sa autoantibodies, an ACPA, (Vossenaar et al., 2004a), and presents in immune complexes in the synovial fluid of RA patients with ACPAs (Van Steendam et al., 2010) and in inflamed synovial tissues (Tilleman et al., 2008). It plays a crucial role in triggering specific autoantibody production and RA pathogenesis (Van Steendam et al., 2011).

The activation of PADls is frequently observed during terminal differentiation and apoptosis. Filaggrin citrullination is presumably an apoptotic event in terminally differentiated keratinocytes (Senshu et al., 1996). In the calcium ionophore-induced apoptosis of macrophages, citrullinated vimentin is found localized around the periphery of the round nucleus, which is an early morphological sign of apoptosis (Asaga et al., 1998). The citrullination of nuclear proteins has also been reported during apoptosis (Mizoguchi et al., 1998). In our previous study, we found that PADI4 overexpression induces apoptosis in HL-60 and Jurkat cells (Liu et al., 2006). In this study, we found that PADI2 overexpression induces apoptosis in activated Jurkat T cells, and we investigated the role of vimentin in PADI2-induced apoptosis.

\section{MATERIALS AND METHODS}

\section{Cell culture and chemical materials}

JK-Tet-On cells were grown in a 90\% RPMI-1640 medium with $10 \%$ fetal bovine serum obtained from Gibco BRL (USA) at a temperature of $37^{\circ} \mathrm{C}$ in a humidified $5 \% \mathrm{CO}_{2}$ environment. Ribonuclease $A$ (RNase $A$ ), acridine orange and doxycycline (Dox), 12-O-tetradecanoylphorbol-13-acetate (TPA), and ionomycin (Ion) were purchased from Sigma (USA).

Human PADI2, mutant PADI2, and vimentin gene subcloning, PADI2 gene knockdown with shRNA, and cell transfection

Human PADI2 and vimentin cDNA were purchased from RZPD Deutsches Ressourcenzentrum für Genomforschung. The D180A mutant of PADI2 (mPADI2) was prepared with the QuikChange site-directed mutagenesis kit (Stratagene, USA), as previously described (Liu et al., 2013), and confirmed by DNA sequencing. The amino acid residue, Asp180, locates in the $\mathrm{N}$-terminal $\mathrm{Ca}^{2+}$-binding site of PADI2 and is essential for catalysis. To amplify PADI2 cDNA, a sense primer (PADI2-s: 19-mer) was used consisting of a $5^{\prime}$ Nott site (underlined) and an 11-nt sequence (nt 65-75): 5'-GCGGCCGCATGCTGCGCG A-3', and an antisense primer (PADI2-as: 18-mer) consisting of a $5^{\prime}$ Sall site (underlined) and a 12-nt sequence (nt 1,4411,430): 5'-GTCGACCACCCCAAAAGA-3'. Vimentin cDNA (encoding 466 amino acids) was amplified using a sense primer (VIM-s: 20-mer) consisting of a $5^{\prime}$ Notl site (underlined) and a 12-nt sequence (nt 292-303): 5'-GCGGCCGCATGTCCACCA GG-3', and an antisense primer (VIM-as: 18-mer) consisting of a $5^{\prime}$ Sall site (underlined) and a 12-nt sequence (nt 1,678-1,689): 5'-GTCGACTTCAAGGTCATC-3'. The polymerase chain reaction (PCR) product was ligated into a $T$ vector, and was transformed in the $E$. coli strain JM109. The bacteria were grown overnight at $37^{\circ} \mathrm{C}$, the plasmids were eluted, and the amplified plasmids were digested with Notl-Sall and subcloned into the
Notl-EcoRI site of pTRE2hyg for the Tet-On system (BD Biosciences Clontech, USA). Only the pTRE2hyg-PADI2, -mPADI2 or -VIM and pTRE2hyg vector were transfected into JK-Tet-On system cells (BD Biosciences, Clontech) by using calcium phosphate-mediated transfection for stable transfection selection. Stably transfected cells were selected with the antibiotic hygromycin $(400 \mu \mathrm{g} / \mathrm{ml})$. After approximately 3 weeks, hygromycin-resistant clones were screened for protein expression by using immunoblotting. In vitro, the promoter induction of the JKTet-On cell system was achieved by adding Dox to the growth medium. PADI2 and luciferase shRNAs were purchased from the Nature RNAi Core Facility, Taipei, Taiwan (NRC). Expression plasmids for PADI2 and luciferase shRNA were made in pLKO.1-puro vector. The targeted shRNA sequences for luciferase and human PADI2 were 5'-GCGGTTGCCAAGAGGTTCC AT-3' and 5'-ACACCGTGATATTCCGGATTG-3', respectively.

Cell viability, apoptotic cell death and acridine orange staining

Living cells were counted using a trypan blue exclusion assay. The cell viability was calculated according to the number of viable cells from the experimental groups divided by those in the control group. To identify apoptotic characteristics, $5 \times 10^{4}$ cells in a 10- $\mu$ l cell suspension were mixed with an equal volume of acridine orange solution $(10 \mu \mathrm{g} / \mathrm{ml})$ in phosphate buffered saline (PBS) on each slide. Green fluorescence was detected and photographed using a fluorescence microscope (Olympus America, USA). Apoptotic cell death was calculated according to the number of fluorescent nuclei (apoptotic cells) divided by the total number of cells, counted in 6 randomly chosen high power fields.

\section{DNA fragmentation analysis}

The cells $\left(5 \times 10^{6}\right)$ were harvested and lysed overnight in a digestion buffer $(0.5 \%$ sarkosyl, $0.5 \mathrm{mg} / \mathrm{ml}$ proteinase $\mathrm{K}, 50 \mathrm{mM}$ Tris- $\mathrm{HCl}, \mathrm{pH} 8.0$, and $10 \mathrm{mM}$ EDTA) at $55^{\circ} \mathrm{C}$. They were subsequently treated with $0.5 \mu \mathrm{g} / \mathrm{ml}$ RNase A for $2 \mathrm{~h}$. The genomic DNA was extracted using phenol-chloroform-isoamyl alcohol, and was analyzed using gel electrophoresis at $50 \mathrm{~V}$ for $90 \mathrm{~min}$ with $2 \%$ agarose. Approximately $20 \mu \mathrm{g}$ of genomic DNA was loaded in each well, visualized under ultraviolet (UV) light, and photographed.

\section{Immunoblotting}

To purify total proteins, the cells were lysed in cold lysis buffer (10\% v/v glycerol, $1 \% \mathrm{v} / \mathrm{v}$ Triton $\mathrm{X}-100,1 \mathrm{mM}$ sodium orthovanadate, $1 \mathrm{mM}$ EGTA, $10 \mathrm{mM} \mathrm{NaF}, 1 \mathrm{mM}$ sodium pyrophosphate, $20 \mathrm{mM}$ Tris, pH 7.9, $100 \mu \mathrm{M} \beta$-glycerophosphate, 137 $\mathrm{mM} \mathrm{NaCl}, 5 \mathrm{mM}$ EDTA, $1 \mathrm{mM}$ PMSF, $10 \mu \mathrm{g} / \mathrm{ml}$ aprotinin, and $10 \mu \mathrm{g} / \mathrm{ml}$ leupeptin), and subsequently homogenized and centrifuged. The supernatants were boiled in a loading buffer and an aliquot corresponding to $100 \mu \mathrm{g}$ of protein separated by SDS-PAGE. After blotting, the membranes were incubated with anti-PADI2 (MDBio), anticaspase-3 (Cell Signaling), anti-PARP (Cell Signaling), antivimentin (Santa Cruz), anticitrulline (Upstate), and anti- $\beta$-actin antibodies (Santa Cruz) for $6 \mathrm{~h}$, and the second antibody labeled with horseradish peroxidase was adjacently incubated for $1 \mathrm{~h}$. The antigen-antibody complexes were visualized using enhanced chemiluminescence (Amersham Pharmacia Biotech, USA).

\section{Immunoprecipitation}

Protein extracts (500 $\mu \mathrm{g}$ per assay) were preabsorbed with $1 \mu \mathrm{g}$ of antivimentin antibodies at $4^{\circ} \mathrm{C}$ for $1 \mathrm{~h}$. Subsequently, protein 


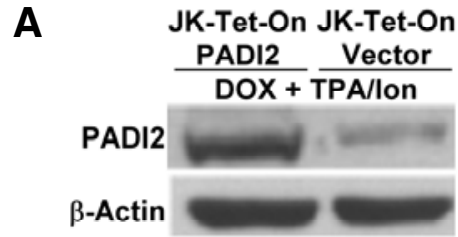

B

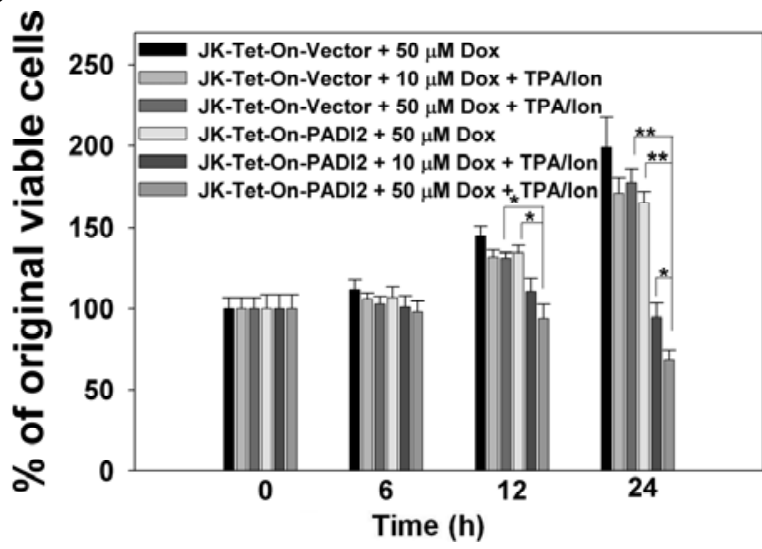

Fig. 1. Overexpression of PADI2 decreases cell viability of activated Jurkat cells. (A) JK-Tet-On-PADI2 and JK-Tet-On-Vector cells were pretreated with $50 \mu \mathrm{M}$ doxycycline (Dox) for $6 \mathrm{~h}$ and treated with $15 \mathrm{ng} / \mathrm{ml}$ 12-O-tetradecanoylphorbol-13-acetate (TPA) plus $1 \mu \mathrm{M}$ ionomycin (Ion) for $6 \mathrm{~h}$. Cells were harvested and total proteins were extracted for immunoblotting with specific antibodies to PADI2 and $\beta$-Actin. (B) JK-Tet-On-PADI2 and JK-Tet-On-Vector cells were pretreated with 10 or $50 \mu \mathrm{M}$ Dox for $6 \mathrm{~h}$, and then treated with or without TPA/lon. Cell viability was determined at indicated time points by trypan blue exclusion assay. ${ }^{\star} P<0.05$ and ${ }^{\star \star} P<0.01$.

$\mathrm{A} / \mathrm{G}$ agarose was added at $4^{\circ} \mathrm{C}$ overnight. After extensive washing, immunoprecipitated proteins were harvested and analyzed using immunoblotting with anticitrulline and antivimentin antibodies.

Fluorescence and differential interference contrast microscopy

After stimulation, JK-Tet-On-Vector and JK-Tet-On-PADI2 cells $\left(1 \times 10^{6}\right)$ were fixed in $2 \%$ paraformaldehyde at room temperature (RT) for 15 min, washed with PBS, and cytospun on coverslips. After blocking with $3 \%$ bovine serum albumin at RT for $2 \mathrm{~h}$, the cells were stained with antivimentin antibodies (1:50) at $4^{\circ} \mathrm{C}$ overnight, washed with PBS, and then incubated with goat antimouse IgG conjugated with rhodamine antibodies (Santa $\mathrm{Cruz}$ ) at $37^{\circ} \mathrm{C}$ for $2 \mathrm{~h}$. The coverslips were mounted onto glass slides and examined using confocal microscopy.

\section{Concentrated protein}

Following incubation, the conditioned media were collected and concentrated using an Amicon Ultra-15 centrifugal filtration device (Millipore), with a molecular weight cutoff set at $30 \mathrm{kDa}$.

\section{Statistical analysis}

Statistical analysis for significant differences between the control and experimental groups was conducted using Student's $t$ test.

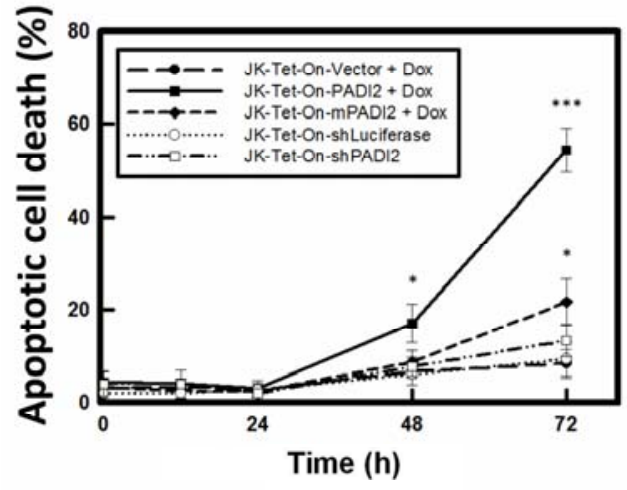

Fig. 2. Apoptotic cell death of JK-Tet-On-PADI2, -mPADI2, -shPA DI2 cells. JK-Tet-On-PADI2, JK-Tet-On-mPADI2 and JK-Tet-OnVector cells were treated with $50 \mu \mathrm{M}$ Dox. Parental JK-Tet-On cells were transfected by PADI2 and Luciferase shRNA (JK-Tet-OnshPADI2 and JK-Tet-On-shLuciferase). Apoptotic cell death (\% fluorescent and condensed nuclei) was determined at indicated time points. ${ }^{*} P<0.05$ and ${ }^{* * *} P<0.001$, compared with JK-Tet-OnVector cells.

\section{RESULTS}

PADI2 overexpression induces apoptosis in activated Jurkat cells

We generated a cell line that overexpressed the PADI2 transgene from a tetracycline-inducible promoter in an otherwise isogenic background (Gossen et al., 1995). In brief, we respectively constructed PADI2, mPADI2 and vector genes into a TetOn expression plasmid (pTRE2hyg), and then transfected them to parental JK-Tet-On cells, namely JK-Tet-On-PADI2, JK-TetOn-mPADI2 and JK-Tet-On-Vector cells. We used a combination of $15 \mathrm{ng} / \mathrm{ml}$ TPA and $1 \mu \mathrm{M}$ lon to induce the activation of Jurkat cells. After treatment with $50 \mu \mathrm{M}$ Dox for $6 \mathrm{~h}$, and then activation by TPA/lon for another $6 \mathrm{~h}$, JK-Tet-On-PADI2 cells expressed greater levels of the PADI2 protein than did the JKTet-On-Vector cells (Fig. 1A).

We observed the effects of PADI2 overexpression on the cell viability of JK-Tet-On-PADI2 cells and their counterparts after they were treated with $10 \mu \mathrm{M}$ or $50 \mu \mathrm{M}$ Dox for $6 \mathrm{~h}$ followed by TPA/lon activation for $24 \mathrm{~h}$. The data revealed that cell proliferation continued in all 3 groups of JK-Tet-On-Vector cells (i.e., + $50 \mu \mathrm{M}$ Dox; + $10 \mu \mathrm{M}$ Dox + TPA/lon; + $50 \mu \mathrm{M}$ Dox + TPA/lon) and the group of JK-Tet-On-PADI2 cells treated with $50 \mu \mathrm{M}$ Dox alone (Fig. 1B). No statistical differences emerged among these 4 groups; in other words, PADI2 overexpression alone did not result in cell death, and did not influence cell proliferation within $24 \mathrm{~h}$. Furthermore, no significant differences emerged for Jurkat cell activation or with varying Dox doses. However, when Jurkat cells with overexpressed PADI2 were activated (JK-TetOn-PADI2 + $10 \mu \mathrm{M}$ Dox + TPA/lon, and JK-Tet-On-PADI2 + 50 $\mu \mathrm{M}$ Dox + TPA/lon), cell viability decreased significantly in a time- and dose-dependent manner within $24 \mathrm{~h}$ (Fig. 1B). In brief, PADI2 overexpression reduced the cell viability of activated Jurkat cells. In addition, we found a significant increase of apoptotic cell death of nonactivated Jurkat cells with PADI2 overexpression at 48 and $72 \mathrm{~h}(17 \%$ and $54 \%$ respectively), compared with JK-Tet-On-Vector cells. PADI2 knockdown did not result in cell death, and mutant PADI2 overexpression had a less apoptotic effect than the wild type (Fig. 2). 
A

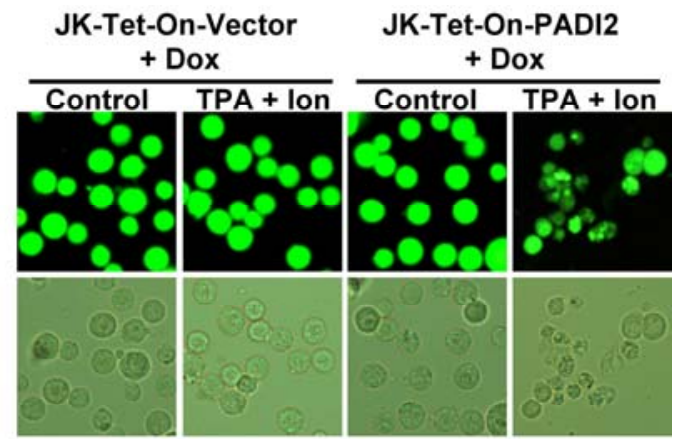

B

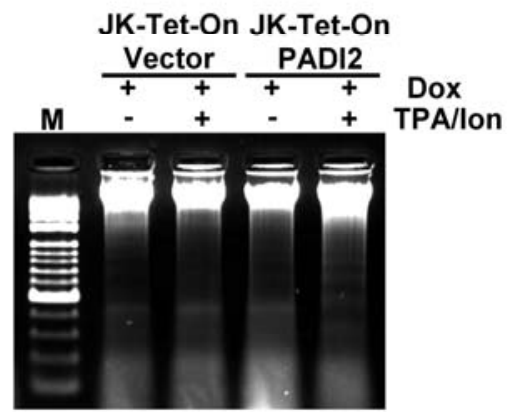

C

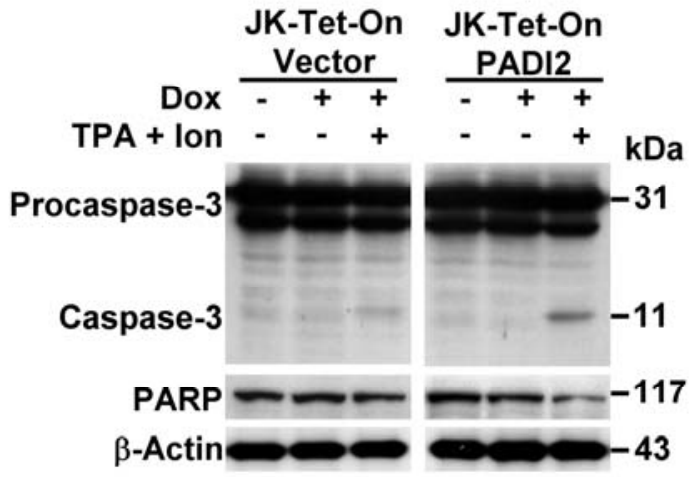

Fig. 3. Overexpression of PADI2 induces apoptosis of activated Jurkat cells. JK-Tet-On-Vector and JK-Tet-On-PADI2 cells were pretreated with $50 \mu \mathrm{M}$ Dox for $6 \mathrm{~h}$ and treated with or without TPA/lon for $24 \mathrm{~h}$. (A) Cells were stained with acridine orange and analyzed by using light microscope and fluorescent microscope. (B) DNA fragmentation was detected by using DNA gel electrophoresis. (C) Total proteins were extracted and analyzed by immunoblotting with anti-caspase-3 and anti-PARP antibodies. Molecular weight markers $(\mathrm{kDa})$ are indicated on the right.

The morphological changes of PADI2-overexpressed and activated Jurkat cells included chromatin condensation, membrane blebbing and shrinkage, and apoptotic bodies (Fig. 3A). Jurkat cells without PADI2 overexpression (JK-Tet-On-Vector + Dox + TPA/lon) or without activation (JK-Tet-On-PADI2 + Dox) exhibited a normal living cell appearance (Fig. 3A). DNA fragmentation (Fig. 3B), caspase-3 activation, and PARP cleavage (Fig. 3 C) presented in the PADI2-overexpressed and -activated Jurkat cells (JK-Tet-On-PADI2 + Dox + TPA/lon), but not in others. All these findings indicated that decreasing cell viability in activated Jurkat cells with PADI2 overexpression is caused by apoptosis.
A

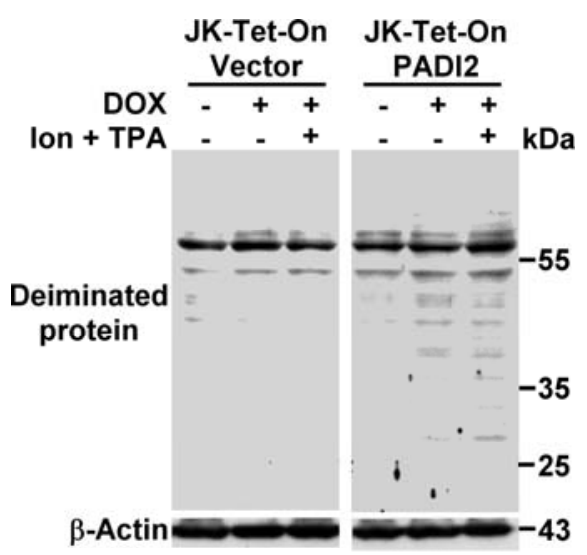

B

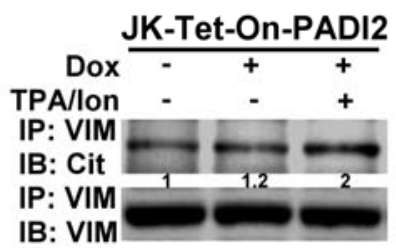

Fig. 4. Jurkat cells with overexpressed PADI2 have more citrullinated proteins and citrullinated vimentin, and further enhanced by cell activation. (A) JK-Tet-On-Vector and JK-Tet-On-PADI2 cells were pretreated with or without Dox $50 \mu \mathrm{M}$ for $6 \mathrm{~h}$, and then treated with or without TPA/lon for $6 \mathrm{~h}$. Cells were harvested and total proteins were extracted for immunoblotting with anti-citrulline antibodies. (B) The cells were lysed, and the lysates were immunoprecipitated with anti-vimentin (VIM) antibodies. The immunoprecipitates were analyzed by immunoblotting with anti-vimentin and anticitrulline (Cit) antibodies. Molecular weight markers $(\mathrm{kDa})$ are indicated on the right.

Enhanced by cell activation, PADI2 overexpression increases the amount of citrullinated proteins, including citrullinated vimentin

To explore the function of overexpressed PADI2, we detected citrullinated proteins and vimentin in JK-Tet-On cells. Intracellular citrullinated proteins were detected using immunoblotting with anticitrulline antibodies. Nonactivated Jurkat cells with PADI2 overexpression (JK-Tet-On-PADI2 + Dox) had more citrullinated proteins than their nonactivated counterparts (JKTet-On-Vector + Dox). When Jurkat cells with PADI2 overexpression were activated (JK-Tet-On-PADI2 + Dox + TPA/lon), they had greater levels of citrullinated proteins than nonactivated Jurkat cells with overexpressed PADI2 (JK-Tet-OnPADI2 + Dox) (Fig. 4A). We immunoprecipitated vimentin, and immunoblotted the precipitants with anticitrulline antibodies. Again, citrullinated vimentin increased 1.2 times in nonactivated PADI2-overexpressed cells (JK-Tet-On-PADI2 + Dox), and 2 times in activated and PADI2 overexpressed cells (JK-Tet-OnPADI2 + Dox + TPA/lon) compared with the control (Fig. 4B). This finding revealed that PADI2 protein, overexpressed by the transfected gene, was functional and deiminated intracellular substrates, including vimentin. The enzyme activity was further enhanced by Jurkat cell activation. 
A

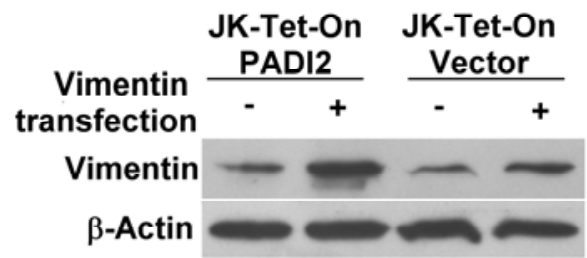

B

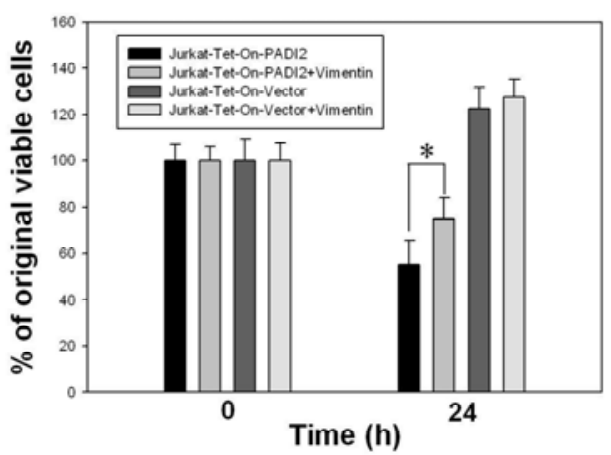

Fig. 5. Overexpression of vimentin attenuates the apoptotic effect of PADI2 on activated Jurkat cells. (A) JK-Tet-On-Vector and JK-TetOn-PADI2 cells were transfected transiently with vimentin for $24 \mathrm{~h}$. Cells were harvested and total proteins were extracted for immunoblotting with anti-vimentin and anti-actin antibodies. (B) Transfected and non-transfected cells were pretreated with $50 \mu \mathrm{M}$ Dox for $6 \mathrm{~h}$, and then treated with TPA/lon for $24 \mathrm{~h}$. Cell viability was determined by trypan blue exclusion assay. ${ }^{*} P<0.05$.

Vimentin is involved in PADI2-induced apoptosis of activated Jurkat cells

To investigate the role of vimentin in PADI2-induced apoptosis, we transiently transfected vimentin into JK-Tet-On PADI2 and JK-Tet-On-Vector cells for $24 \mathrm{~h}$ to force-express a greater amount of normal vimentin in cells (Fig. 5A). Vimentin overexpression did not alter the cell viability or proliferation of activated Jurkat cells without PADI2 overexpression (Fig. 5B). In activated Jurkat cells with PADI2 overexpression, however, cell viability increased significantly in cells with force-expressed vimentin compared with those without. Vimentin overexpression rescued a portion of Jurkat cells from apoptosis, which was induced by PADI2 overexpression (Fig. 5B); that is, vimentin was involved in PADI2-induced apoptosis in activated Jurkat cells.

Vimentin is expressed on cell surfaces and secreted by activated Jurkat cells with overexpressed PADI2

Mor-Vaknin et al. (2003) reported that vimentin is secreted by activated macrophages, and Boilard et al. (2003) indicated that it is partially exposed on the surface of apoptotic T cells. To investigate whether vimentin was expressed on cell surfaces and secreted during PADI2-induced apoptosis, we pretreated JK-Tet-On-Vector and JK-Tet-On-PADI2 cells with $50 \mu \mathrm{M}$ Dox for $6 \mathrm{~h}$, treated them with TPA/lon, collected the supernatants at $12 \mathrm{~h}$ and $24 \mathrm{~h}$, and harvested all the cells at $24 \mathrm{~h}$.

PADI2-overexpressed and -activated Jurkat cells (JK-Tet-OnPADI2+Dox+TPA/lon) revealed greater levels of immunostaining compared with their counterparts when treated with the antivimentin antibody combined with the rhodamine-conjugated antimouse secondary antibody (Fig. 6). We merged the photographs

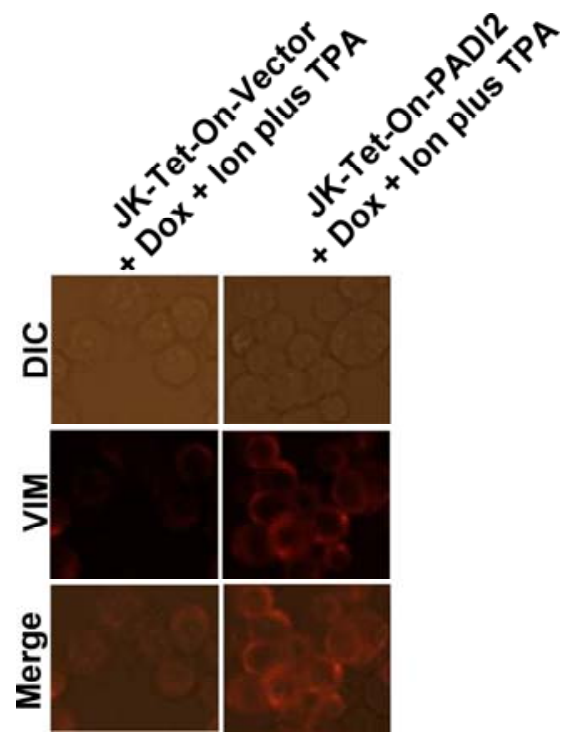

Fig. 6. Vimentin is expressed on cell surfaces of activated Jurkat cells with overexpressed PADI2. JK-Tet-On-Vector and JK-Tet-OnPADI2 cells were pretreated with $50 \mu \mathrm{M}$ Dox for $6 \mathrm{~h}$, and then treated with TPA/lon for $24 \mathrm{~h}$. Cells were stained with anti-vimentin (VIM) antibody, followed by rhodamine-conjugated anti-mouse secondary antibody (red), observed and photographed by differential interference contrast microscopy (DIC) and fluorescence microscopy. Confocal merge showed the location of vimentin.

obtained using differential interference contrast microscopy and fluorescence microscopy (VIM), and found that the binding sites of the antivimentin antibody were on the cell surfaces. These findings showed that vimentin was expressed on the cell surfaces of PADI2-overexpressed and -activated Jurkat cells while they were undergoing apoptosis.

We concentrated the supernatants by using a centrifugal filtration device with the molecular weight cutoff set at $30 \mathrm{kDa}$, and then analyzed them using immunoblotting with antivimentin antibodies. After activating JK-Tet-On-Vector cells, we found a greater amount of vimentin in the supernatant collected at $24 \mathrm{~h}$ than in that collected at $12 \mathrm{~h}$ (Fig. 7). At $12 \mathrm{~h}$, the supernatant of PADI2-overexpressed and -activated Jurkat cells had more vimentin than the supernatant of their counterparts (Fig. 7); in other words, T-cell activation and PADI2 overexpression promoted the secretion of vimentin. However, at $24 \mathrm{~h}$, the supernatant of PADI2-overexpressed and -activated Jurkat cells had less vimentin.

\section{DISCUSSION}

Triggering the antigen-specific T-cell receptor (TcR)/CD3 complex initiates a cascade of signal transductions across the cell membrane, resulting in activation and proliferation. One signal transduction pathway involves a phospholipase $C$ species that hydrolyzes phosphatidylinositol 4,5-bisphosphate and generates 2 second messengers, diacylglycerol and inositol 1,4,5trisphosphate (reviewed by Altman et al., 1990). Diacylglycerol activates protein kinase C (PKC) (Isakov et al., 1987; Nishizuka, 1988). Inositol 1,4,5-trisphosphate releases $\mathrm{Ca}^{2+}$ from the 


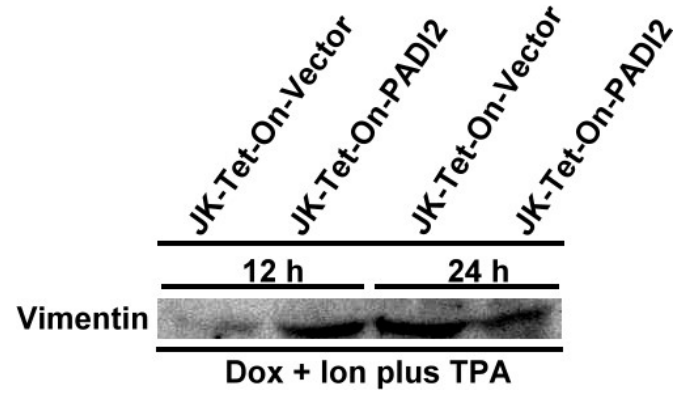

Fig. 7. Vimentin presents in the supernatants of activated Jurkat cells. JK-Tet-On-Vector and JK-Tet-On-PADI2 cells were pretreated with $50 \mu \mathrm{M}$ Dox for $6 \mathrm{~h}$, and then treated with TPA/lon. The supernatants were collected at $12 \mathrm{~h}$ and $24 \mathrm{~h}$, concentrated and then analyzed by immunoblotting with anti-vimentin antibody.

endoplasmic reticulum, resulting in an increase in the intracellular concentration of free $\mathrm{Ca}^{2+}$ (Imboden and Stobo, 1985). Therefore, a TcR/CD3-induced cellular response involves an increase in cytosolic calcium concentration and protein kinase $C$ activation. The phorbol ester TPA, which is structurally related to diacylglycerol, directly binds to, and activates, PKC (Castagna et al., 1982). The calcium ionophore lon stimulates the $\mathrm{Ca}^{2+}$ influx (Liu and Hermann, 1978). Thus, the combination of TPA and lon mimics the antigen-induced signal at the onset of lymphocyte activation (Altman et al., 1992; Truneh et al., 1985).

PADI2 and PADI4 are the only 2 isotypes expressed in the synovial tissue of patients with RA and those with other arthritides. Inflammatory cells, including lymphocytes and monocytes, infiltrate the synovial tissue, and are a major source of PADI2 and PADI4. The expression levels of PADI2 and PADI4 are correlated with the intensity of inflammation (Foulquier et al., 2007). In our study, Jurkat T lymphocytes exhibited endogenous PADI2 protein. Using a combination of TPA and Ion to activate Jurkat cells, we found that activated Jurkat cells expressed greater levels of PADI2 and exhibited greater PADI enzyme activity (data not shown). The expression of endogenous PADI2 may be transcriptionally regulated by TPA, because 4 plausible Sp1 binding sites are located in the PADI2 promoter region (Dong et al., 2005; Kim et al., 2004). The PADI2-Tet-On system functioned successfully to express exogenous PADI2 in activated Jurkat cells (Fig. 1A). Moreover, overexpressed PADI2 displayed enzyme activity, which we inferred by noting the production of more citrullinated proteins (Fig. 4).

PADIs rely strongly on the presence of $\mathrm{Ca}^{2+}$ for activity. A typical concentration of cytosolic $\mathrm{Ca}^{2+}$ is approximately $10^{-7} \mathrm{M}$, which is too low for PADI2 activity (Asaga et al., 1998; Vossenaar et al., 2004b). The minimum $\mathrm{Ca}^{2+}$ concentration required for PADI2 activity is approximately 100 -fold higher than that required for normal cytosolic $\mathrm{Ca}^{2+}$ (Takahara et al., 1986). Although nonactivated Jurkat cells expressed endogenous and exogenous PADI2, most of the enzymes were inactivated because of the low cytosolic $\mathrm{Ca}^{2+}$ concentration. When the Jurkat cells were activated, raising the cytosolic $\mathrm{Ca}^{2+}$ concentration, they activated PADI2 to deiminate intracellular substrates. In addition, we observed that activated cells with overexpressed PADI2 presented the typical manifestations of apoptosis. This paper is the first to provide direct evidence that PADI2 overexpression induces apoptosis in activated Jurkat cells.

Citrullinated proteins are detected in the inflammatory syn- ovial fluid both in patients with RA and in patients with spondyloarthritis (Kinloch et al., 2008). However, the presentation of intracellular citrullinated proteins in the synovium is specific for RA (Baeten et al., 2001), and is colocalized with PADI2 (De Rycke, 2005). Numerous well-known or suspected intracellular substrates of PADI2 and PADI4 exist, such as vimentin (Asaga et al., 1998; Vossenaar et al., 2004b), histones (Nakashima et al., 2002), nucleophosmin/B23 (Hagiwara et al., 2002), the Factin capping protein alpha 1 subunit, cathepsin $\mathrm{D}$, beta-actin (Matsuo et al., 2006), aldolase, alpha-enolase, phosphoglycerate kinase 1 , calreticulin, the $60-\mathrm{kDa}$ heat shock protein, far upstream element-binding proteins 1 and 2 (Goëb et al., 2009), glucose-6-phosphate isomerase (Umeda et al., 2013), and IKKY (Lee et al., 2010). Among these, vimentin is the major citrullinated protein in human monocytic leukemia THP-1 cells during macrophage differentiation (Hojo-Nakashima et al., 2009). Our study revealed that vimentin is a target protein of PADI2 in Jurkat cells during activation and apoptosis, as reported in mouse peritoneal and human macrophages (Asaga et al., 1998; Vossenaar et al., 2004b), as well as in human monocytic leukemia THP-1 cells (Hojo-Nakashima et al., 2009). In addition to its mechanical and structural properties, vimentin plays a crucial role in adhesion, migration, survival, and cell signaling (reviewed in Hendrix et al., 1996; Wang and Stamenovic, 2002). The exact mechanisms of vimentin functionality have not been fully elucidated, and have instead been attributed to the dynamic disassembly/assembly and spatial reorganization regulated by phosphorylation (Inagaki et al., 1989; Ivaska et al., 2007). PADI2 predominantly deiminates the nonalphahelical $\mathrm{N}$-terminal head domain of vimentin, which is rich in arginine, serine, and threonine, and is prone to phosphorylation (Inagaki et al., 1989; Ivaska et al., 2007). Vimentin citrullination results in the disassembly of vimentin filaments, the prevention of assembly (Inagaki et al., 1989), loss of functions, and the transformation of the fine network across the whole cell into amorphous clusters located around the nucleus (Asaga et al., 1998; Hojo-Nakashima et al., 2009).

The role of vimentin in apoptosis has been demonstrated in withaferin A (WFA)-induced apoptosis (Lahat et al., 2010). WFA, a naturally derived bioactive compound, binds to vimentin by covalently modifying its cysteine residue, which is present in the highly conserved alpha-helical coiled coil 2B domain (Bargagna-Mohan et al., 2007). It transforms vimentin filaments to punctate cytoplasmic aggregates that colocalize vimentin and F-actin (Bargagna-Mohan et al., 2007). Caspases rapidly degrade vimentin after exposure to different proapoptotic stimuli, including through ionizing radiation, and Fas, TRAIL, tumor necrosis factor $\alpha(T N F \alpha)$, and tamoxifen administration (Hashimoto et al., 1998; Morishima, 1999; Prasad et al., 1998). The inhibition of vimentin degradation (by caspase inhibitors or the overexpression of caspase-resistant vimentin) abrogates WFAinduced apoptosis (Lahat et al., 2010). If vimentin is degraded by activated caspases, potential proapoptotic fragments are released and substantially enhance apoptosis (Byun et al., 2001). A positive feedback loop forms, whereby activated caspases degrade vimentin, and these degraded fragments consequently activate caspases to amplify apoptosis.

Our findings showed that vimentin overexpression at least partially rescued PADI2-overexpressed and -activated Jurkat cells. Cells with greater levels of vimentin exhibited a greater ability to withstand the impact of citrullination, and avoid apoptosis; in other words, vimentin is involved in the mechanism of PADI2-induced apoptosis. The overexpression of PADI2 increased the amount of citrullinated proteins, including citrulli- 
nated vimentin. Citrullinated vimentin lost its normal functions, and was probably unfolded and degraded by calpains. Overall, the inferential mechanism of PADI2-induced apoptosis may contribute to the initiation of apoptotic signals through the loss of normal vimentin functions, the unfolded protein response or pro-apoptotic vimentin fragments.

Although vimentin is an intracellular protein, it is expressed on cell membranes under certain distinctive conditions such as by platelets and macrophages during activation (Mor-Vaknin et al., 2003; Podor et al., 2002) and by lymphocytes and neutrophils during apoptosis (Boilard et al., 2003; Moisan and Girard, 2006). Vimentin is partially exposed on the surface of apoptotic T cells, and binds human group IIA phospholipase A2 (PLA2) through its rod domain in a calcium-independent manner (Boilard et al., 2003). The binding of these 2 proteins enhanced PLA2 activity, suggesting that vimentin may play a role in PLA2-mediated cellular arachidonic acid release. Vimentin is expressed on the surface of apoptotic neutrophils (Moisan and Girard, 2006), and is cleaved by the neutrophil-specific protease membrane-type 6 matrix metalloproteinase (Starr et al., 2012). Cleaved vimentin on the cell surface potently promotes phagocytosis, and functions as an "eat me" signal to macrophages. This shows another role of vimentin in increasing the phagocytic removal of neutrophils to resolve inflammation (Starr et al., 2012). Compatible with previous studies, we found that vimentin was expressed on the cell surface of PADI2overexpressed and -activated Jurkat cells while they were undergoing apoptosis. However, our data cannot provide an answer as to whether PADI2 contributes to this cell-surface expression or whether expressed vimentin is citrullinated. Further in-depth investigation is required to answer these questions. Moreover, vimentin secretion can be triggered by activated macrophages (Mor-Vaknin et al., 2003). Vimentin secretion is blocked by antiinflammatory cytokine interleukin-10, but is triggered by proinflammatory cytokine TNF $\alpha$. Extracellular vimentin is involved in bacterial killing and the generation of oxidative metabolites (Mor-Vaknin et al., 2003), and is a chemoattractant for monocytes (Starr et al., 2012). We found that vimentin was secreted by activated Jurkat cells, which were further enhanced by PADI2 overexpression in the initial $12 \mathrm{~h}$ of activation. However, the amount of vimentin in the supernatant of PADI2overexpressed and -activated Jurkat cells decreased $24 \mathrm{~h}$ after activation. We found citrullinated vimentin in all of the supernatants (data not shown). However, we failed to demonstrate an explainable and consistent result, possibly because numerous proteases are present in the intracellular and extracellular spaces, such as caspases, calpains, and matrix metalloproteinases, which are activated during cell activation or apoptosis, and degrade vimentin.

T lymphocytes are central to the specific immune response, and following their activation, they are eliminated by a Fasmediated cell death program (Savill, 1997). The presence of activated T cells in the inflamed synovium of patients with RA is a primary indicator of the disease, and apoptosis is a crucial mechanism for their elimination and the eventual resolution of inflammation (Savill, 1997; Savill et al., 1989). It is typically believed that apoptotic immune cells can undergo secondary necrosis and release their contents in the extracellular space when they are overly abundant or cannot be cleared normally. In an extracellular space with a high $\mathrm{Ca}^{2+}$ concentration, PADI2 and PADI4 citrullinate extracellular proteins, which are then exposed to the immune system and elicit the formation of $A C$ PAs (van Venrooij et al., 2011). Through artificially highlighting PADI2 and vimentin, we demonstrated that PADI2 and vimen- tin participate in the apoptotic mechanisms of active T lymphocytes. Even in the normal course of T-cell apoptosis, the secretion and surface expression of vimentin are possible ways of autoantigen presentation to the immune system.

\section{ACKNOWLEDGMENTS}

This work was supported by the National Science Council, ROC (NSC102-2321-B-005-008 to H.-C. Hung; NSC 1022320-B-040 -018 -MY3 to G.-Y. Liu) and in part by the Ministry of Education, Taiwan, R.O.C. under the ATU plan.

\section{REFERENCES}

Altman, A., Coggeshall, K.M., and Mustelin, T. (1990). Molecular events mediating T cell activation. Adv. Immunol. 48, 227-360.

Altman, A., Mally, M.I., and Isakov, N. (1992). Phorbol ester synergizes with $\mathrm{Ca}^{2+}$ ionophore in activation of protein kinase $\mathrm{C}$ (PKC) alpha and PKC beta isoenzymes in human T cells and in induction of related cellular functions. Immunology 76, 465-471.

Asaga, H., Yamada, M., and Senshu, T. (1998). Selective deimination of vimentin in calcium ionophore-induced apoptosis of mouse peritoneal macrophages. Biochem. Biophys. Res. Commun. 243, 641-646.

Asaga, H., Nakashima, K., Senshu, T., Ishigami, A., and Yamada, M. (2001). Immunocytochemical localization of peptidylarginine deiminase in human eosinophils and neutrophils. J. Leukoc. Biol. 70, 46-51.

Baeten, D., Peene, I., Union, A., Meheus, L., Sebbag, M., Serre, G. Veys, E.M., and De Keyser, F. (2001). Specific presence of intracellular citrullinated proteins in rheumatoid arthritis synovium: relevance to antifilaggrin autoantibodies. Arthritis Rheum. 44, 2255-2262.

Bargagna-Mohan, P., Hamza, A., Kim, Y.E., Khuan Abby Ho, Y., Mor-Vaknin, N., Wendschlag, N., Liu, J., Evans, R.M., Markovitz, D.M., Zhan, C.G., et al. (2007). The tumor inhibitor and antiangiogenic agent withaferin $A$ targets the intermediate filament protein vimentin. Chem. Biol. 14, 623-634.

Boilard, E., Bourgoin, S.G., Bernatchez, C., and Surette, M.E. (2003) Identification of an autoantigen on the surface of apoptotic human T cells as a new protein interacting with inflammatory group IIA phospholipase A2. Blood 102, 2901-2909.

Byun, Y., Chen, F., Chang, R., Trivedi, M., Green, K.J., and Cryns, V.L. (2001). Caspase cleavage of vimentin disrupts intermediate filaments and promotes apoptosis. Cell Death Differ. 8, 443-450.

Castagna, M., Takai, Y., Kaibuchi, K., Sano, K., Kikkawa, U., and Nishizuka, Y. (1982). Direct activation of calcium-activated, phospholipid-dependent protein kinase by tumor-promoting phorbol esters. J. Biol. Chem. 257, 7847-7851.

De Rycke, L., Nicholas, A.P., Cantaert, T., Kruithof, E., Echols, J.D., Vandekerckhove, B., Veys, E.M., De Keyser, F., and Baeten, D. (2005). Synovial intracellular citrullinated proteins colocalizing with peptidyl arginine deiminase as pathophysiologically relevant antigenic determinants of rheumatoid arthritis-specific humoral autoimmunity. Arthritis Rheum. 52, 2323-2330.

Dong, S., Kojima, T., Shiraiwa, M., Méchin, M.C., Chavanas, S., Serre, G., Simon, M., Kawada, A., and Takahara, H. (2005). Regulation of the expression of peptidylarginine deiminase type II gene (PADI2) in human keratinocytes involves Sp1 and Sp3 transcription factors. J. Invest. Dermatol. 124, 1026-1033.

Foulquier, C., Sebbag, M., Clavel, C., Chapuy-Regaud, S., Al Badine, R., Méchin, M.C., Vincent, C., Nachat, R., Yamada, M., Takahara, $H_{\text {., }}$ et al. (2007). Peptidyl arginine deiminase type 2 (PAD-2) and PAD-4 but not PAD-1, PAD-3, and PAD-6 are expressed in rheumatoid arthritis synovium in close association with tissue inflammation. Arthritis Rheum. 56, 3541-3553.

Goëb, V., Thomas-L'Otellier, M., Daveau, R., Charlionet, R., Fardellone, P., Le Loët, X., Tron, F., Gilbert, D., and Vittecoq, O. (2009). Candidate autoantigens identified by mass spectrometry in early rheumatoid arthritis are chaperones and citrullinated glycolytic enzymes. Arthritis Res. Ther. 11, R38.

Gossen, M., Freundlieb, S., Bender, G., Müller, G., Hillen, W., and Bujard, H. (1995). Transcriptional activation by tetracyclines in mammalian cells. Science 268, 1766-1769. 
Hagiwara, T., Nakashima, K., Hirano, H., Senshu, T., and Yamada M. (2002). Deimination of arginine residues in nucleophosmin/B23 and histones in HL-60 granulocytes. Biochem. Biophys. Res. Commun. 290, 979-983.

Hashimoto, M., Inoue, S., Ogawa, S., Conrad, C., Muramatsu, M., Shackelford, D., and Masliah, E. (1998). Rapid fragmentation of vimentin in human skin fibroblasts exposed to tamoxifen: a possible involvement of caspase-3. Biochem. Biophys. Res. Commun. 247, 401-406.

Hay, E.D. (1989). Extracellular matrix, cell skeletons, and embryonic development. Am. J. Med. Genet. 34, 14-29.

Hendrix, M.J., Seftor, E.A., Chu, Y.W., Trevor, K.T., and Seftor, R.E. (1996). Role of intermediate filaments in migration, invasion and metastasis. Cancer Metastasis Rev. 15, 507-525.

Hojo-Nakashima, I., Sato, R., Nakashima, K., Hagiwara, T., and Yamada, M. (2009). Dynamic expression of peptidylarginine deiminase 2 in human monocytic leukaemia THP-1 cells during macrophage differentiation. J. Biochem. 146, 471-479.

Imboden, J.B., and Stobo, J.D. (1985). Transmembrane signalling by the $T$ cell antigen receptor. Perturbation of the T3-antigen receptor complex generates inositol phosphates and releases calcium ions from intracellular stores. J. Exp. Med. 161, 446-456.

Inagaki, M., Takahara, H., Nishi, Y., Sugawara, K., and Sato, C. (1989). $\mathrm{Ca}^{2+}$-dependent deimination-induced disassembly of intermediate filaments involves specific modification of the aminoterminal head domain. J. Biol. Chem. 264, 18119-18127.

Isakov, N., Mally, M.I., Scholz, W., and Altman, A. (1987). Tlymphocyte activation: the role of protein kinase $\mathrm{C}$ and the bifurcating inositol phospholipid signal transduction pathway. Immunol. Rev. 95, 89-111.

Ishigami, A., Ohsawa, T., Hiratsuka, M., Taguchi, H., Kobayashi, S., Saito, Y., Murayama, S., Asaga, H., Toda, T., Kimura, N., et al. (2005). Abnormal accumulation of citrullinated proteins catalyzed by peptidylarginine deiminase in hippocampal extracts from patients with Alzheimer's disease. J. Neurosci. Res. 80, 120-128

Ivaska, J., Pallari, H.M., Nevo, J., and Eriksson, J.E. (2007). Novel functions of vimentin in cell adhesion, migration, and signaling. Exp. Cell Res. 313, 2050-2062.

Jang, B., Jeon, Y.C., Choi, J.K., Park, M., Kim, J.I., Ishigami, A Maruyama, N., Carp, R.I., Kim, Y.S., and Choi, E.K. (2012) Peptidylarginine deiminase modulates the physiological roles of enolase via citrullination: links between altered multifunction of enolase and neurodegenerative diseases. Biochem. J. 445, 183-192.

Kamata, Y., Taniguchi, A., Yamamoto, M., Nomura, J., Ishihara, K., Takahara, H., Hibino, T., and Takeda, A. (2009). Neutral cysteine protease bleomycin hydrolase is essential for the breakdown of deiminated filaggrin into amino acids. J. Biol. Chem. 284, 12829-12836

Kim, E., Muga, S.J., and Fischer, S.M. (2004). Identification and characterization of a phorbol ester-responsive element in the murine 8S-lipoxygenase gene. J. Biol. Chem. 279, 11188-11197.

Kinloch, A., Lundberg, K., Wait, R., Wegner, N., Lim, N.H., Zendman, A.J., Saxne, T., Malmström, V., and Venables, P.J. (2008). Synovial fluid is a site of citrullination of autoantigens in inflammatory arthritis. Arthritis Rheum. 58, 2287-2295.

Lahat, G., Zhu, Q.S., Huang, K.L., Wang, S., Bolshakov, S., Liu, J., Torres, K., Langley, R.R., Lazar, A.J., Hung, M.C., et al. (2010). Vimentin is a novel anti-cancer therapeutic target; insights from in vitro and in vivo mice xenograft studies. PLoS One 5, e10105.

Lee, H.J., Joo, M., Abdolrasulnia, R., Young, D.G., Choi, I., Ware, L.B., Blackwell, T.S., and Christman, B.W. (2010). Peptidylarginine deiminase 2 suppresses inhibitory \{kappa\}B kinase activity in lipopolysaccharide-stimulated RAW 264.7 macrophages. J. Biol. Chem. 285, 39655-39662.

Liu, C., and Hermann, T.E. (1978). Characterization of ionomycin as a calcium ionophore. J. Biol. Chem. 253, 5892-5894.

Liu, G.Y., Liao, Y.F., Chang, W.H., Liu, C.C., Hsieh, M.C., Hsu, P.C., Tsay, G.J., and Hung, H.C. (2006). Overexpression of peptidylarginine deiminase IV features in apoptosis of haematopoietic cells. Apoptosis 11, 183-196.

Liu, Y.L., Tsai, I.C., Chang, C.W., Liao, Y.F., Liu, G.Y., and Hung, H.C. (2013). Functional roles of the non-catalytic calciumbinding sites in the $\mathrm{N}$-terminal domain of human peptidylarginine deiminase 4. PLoS One 8, e51660.
Lundkvist, A., Reichenbach, A., Betsholtz, C., Carmeliet, P., Wolburg, H., and Pekny, M. (2004). Under stress, the absence of intermediate filaments from Müller cells in the retina has structural and functional consequences. J. Cell Sci. 117, 3481-3488.

Matsuo, K., Xiang, Y., Nakamura, H., Masuko, K., Yudoh, K., Noyori, K., Nishioka, K., Saito, T., and Kato, T. (2006). Identification of novel citrullinated autoantigens of synovium in rheumatoid arthritis using a proteomic approach. Arthritis Res. Ther. 8, R175.

Mizoguchi, M., Manabe, M., Kawamura, Y., Kondo, Y., Ishidoh, K., Kominami, E., Watanabe, K., Asaga, H., Senshu, T., and Ogawa, H. (1998). Deimination of 70-kD nuclear protein during epidermal apoptotic events in vitro. J. Histochem. Cytochem. 46, 1303-1309.

Moisan, E., and Girard, D. (2006). Cell surface expression of intermediate filament proteins vimentin and lamin B1 in human neutrophil spontaneous apoptosis. J. Leukoc. Biol. 79, 489-498.

Morishima, N. (1999). Changes in nuclear morphology during apoptosis correlate with vimentin cleavage by different caspases located either upstream or downstream of Bcl-2 action. Genes Cells 4, 401-414.

Mor-Vaknin, N., Punturieri, A., Sitwala, K., and Markovitz, D.M. (2003). Vimentin is secreted by activated macrophages. Nat. Cell Biol. 5, 59-63.

Moscarello, M.A., Mastronardi, F.G., and Wood, D.D. (2007). The role of citrullinated proteins suggests a novel mechanism in the pathogenesis of multiple sclerosis. Neurochem. Res. 32, 251 256.

Nakashima, K., Hagiwara, T., Ishigami, A., Nagata, S., Asaga, H., Kuramoto, M., Senshu, T., and Yamada, M. (1999). Molecular characterization of peptidylarginine deiminase in $\mathrm{HL}-60$ cells induced by retinoic acid and 1alpha,25-dihydroxyvitamin $\mathrm{D}(3)$. J. Biol. Chem. 274, 27786-27792.

Nakashima, K., Hagiwara, T., and Yamada, M. (2002). Nuclear localization of peptidylarginine deiminase $\mathrm{V}$ and histone deimination in granulocytes. J. Biol. Chem. 277, 49562-49568.

Nicholas, A.P. (2011). Dual immunofluorescence study of citrullinated proteins in Parkinson diseased substantia nigra. Neurosci. Lett. 495, 26-29.

Nicholas, A.P., Sambandam, T., Echols, J.D., and Tourtellotte, W.W. (2004). Increased citrullinated glial fibrillary acidic protein in secondary progressive multiple sclerosis. J. Comp. Neurol. 473, 128-136.

Nishizuka, Y. (1988). The molecular heterogeneity of protein kinase C and its implications for cellular regulation. Nature 334, 661-665.

Podor, T.J., Singh, D., Chindemi, P., Foulon, D.M., McKelvie, R., Weitz, J.I., Austin, R., Boudreau, G., and Davies, R. (2002). Vimentin exposed on activated platelets and platelet microparticles localizes vitronectin and plasminogen activator inhibitor complexes on their surface. J. Biol. Chem. 277, 7529-7539.

Prasad, S.C., Thraves, P.J., Kuettel, M.R., Srinivasarao, G.Y., Dritschilo, A and Soldatenkov, V.A. (1998). Apoptosis-associated proteolysis of vimentin in human prostate epithelial tumor cells. Biochem. Biophys. Res. Commun. 249, 332-338.

Pritzker, L.B., Joshi, S., Gowan, J.J., Harauz, G., and Moscarello, M.A. (2000). Deimination of myelin basic protein. 1. Effect of deimination of arginyl residues of myelin basic protein on its structure and susceptibility to digestion by cathepsin D. Biochemistry 39, 5374-5381.

Savill, J. (1997). Apoptosis in resolution of inflammation. J. Leukoc. Biol. 61, 375-380

Savill, J.S., Wyllie, A.H., Henson, J.E., Walport, M.J., Henson, P.M., and Haslett, C. (1989). Macrophage phagocytosis of aging neutrophils in inflammation. Programmed cell death in the neutrophil leads to its recognition by macrophages. J. Clin. Invest. 83,865 875

Senshu, T., Kan, S., Ogawa, H., Manabe, M., and Asaga, H. (1996). Preferential deimination of keratin $\mathrm{K} 1$ and filaggrin during the terminal differentiation of human epidermis. Biochem. Biophys. Res. Commun. 225, 712-719.

Starr, A.E., Bellac, C.L., Dufour, A., Goebeler, V., and Overall, C.M (2012). Biochemical characterization and N-terminomics analysis of leukolysin, the membrane-type 6 matrix metalloprotease (MMP25): chemokine and vimentin cleavages enhance cell migration and macrophage phagocytic activities. J. Biol. Chem. 287, 13382-13395

Takahara, H., Okamoto, H., and Sugawara, K. (1986). Affinity 
chromatography of peptidylarginine deiminase from rabbit skeletal muscle on a column of soybean trypsin inhibitor (Kunitz)Sepharose. J. Biochem. 99, 1417-1424.

Tarcsa, E., Marekov, L.N., Mei, G., Melino, G., Lee, S.C., and Steinert, P.M. (1996). Protein unfolding by peptidylarginine deiminase. Substrate specificity and structural relationships of the natural substrates trichohyalin and filaggrin. J. Biol. Chem. 271, 30709-30716.

Tilleman, K., Van Steendam, K., Cantaert, T., De Keyser, F., Elewaut, D., and Deforce, D. (2008). Synovial detection and autoantibody reactivity of processed citrullinated isoforms of vimentin in inflammatory arthritides. Rheumatology 47, 597-604

Truneh, A., Albert, F., Golstein, P., and Schmitt-Verhulst, A.M (1985). Early steps of lymphocyte activation bypassed by synergy between calcium ionophores and phorbol ester. Nature 313 318-320.

Umeda, N., Matsumoto, I., Ito, I., Kawasaki, A., Tanaka, Y., Inoue, A., Tsuboi, H., Suzuki, T., Hayashi, T., Ito, S., et al. (2013) Anticitrullinated glucose-6-phosphate isomerase peptide antibodies in patients with rheumatoid arthritis are associated with HLADRB1 shared epitope alleles and disease activity. Clin. Exp. Immunol. 172, 44-53.

Van Steendam, K., Tilleman, K., De Ceuleneer, M., De Keyser, F., Elewaut, D., and Deforce, D. (2010). Citrullinated vimentin as an important antigen in immune complexes from synovial fluid of rheumatoid arthritis patients with antibodies against citrullinated proteins. Arthritis Res. Ther. 12, R132.
Van Steendam, K., Tilleman, K., and Deforce, D. (2011). The relevance of citrullinated vimentin in the production of antibodies against citrullinated proteins and the pathogenesis of rheumato id arthritis. Rheumatology 50, 830-837.

Van Venrooij, W.J., van Beers, J.J., and Pruijn, G.J. (2011). AntiCCP antibodies: the past, the present and the future. Nat. Rev. Rheumatol. 7, 391-398.

Vossenaar, E.R., Zendman, A.J., van Venrooij, W.J., and Pruijn, G.J. (2003). PAD, a growing family of citrullinating enzymes: genes, features and involvement in disease. Bioessays 25, 1106-1118.

Vossenaar, E.R., Després, N., Lapointe, E., van der Heijden, A., Lora, M., Senshu, T., van Venrooij, W.J., and Ménard, H.A. (2004a) Rheumatoid arthritis specific anti-Sa antibodies target citrullinated vimentin. Arthritis Res. Ther. 6, R142-150

Vossenaar, E.R., Radstake, T.R., van der Heijden, A., van Mansum, M.A., Dieteren, C., de Rooij, D.J., Barrera, P., Zendman, A.J., and van Venrooij, W.J. (2004b). Expression and activity of citrullinating peptidylarginine deiminase enzymes in monocytes and macrophages. Ann. Rheum. Dis. 63, 373-381.

Wang, N., and Stamenovic, D. (2002). Mechanics of vimentin intermediate filaments. J. Muscle Res. Cell Motil. 23, 535-540.

Zhou, Z., and Ménard, H.A. (2002). Autoantigenic posttranslational modifications of proteins: does it apply to rheumatoid arthritis? Curr. Opin. Rheumatol. 14, 250-253. 